\title{
Dialogization and Implicit Information in an Agent Communicational Model
}

\author{
Karim Bouzouba ${ }^{1}$, Jamal Bentahar ${ }^{2}$, and Bernard Moulin ${ }^{2}$ \\ ${ }^{1}$ IERA, Mohamed V Souissi University, \\ BP 6216, Rabat, Morocco \\ karim.bouzoubaa@iera.ac.ma \\ ${ }^{2}$ Laval University, Department of Computer Science and Software Engineering, \\ Ste-Foy, QC, G1K 7P4, Canada \\ jamal.bentahar.1@ulaval.ca \\ bernard.moulin@ift.ulaval.ca
}

\begin{abstract}
In this paper we propose a computational model for human-agent and agent-agent conversation. This model has two fundamental characteristics: (1) it takes into account the implicit aspects of conversations by dealing with the non literal level of speech acts; (2) it models the dialogization process. Theoretically, our model uses a public approach based on social commitments and on what we call communicational states. In addition, we consider communication as a negotiation process formed by a set of initiative/reactive dialogue games. The paper also presents an implementation of our model in a multi-agent system called POSTAGE.
\end{abstract}

\section{Introduction}

For almost a decade, industry and researchers have been seriously considering applications involving "conversational interfaces" instead of the classical graphical user interfaces [18, 22]. A conversational interface attempts to leverage natural aspects of human dialogue and social interaction, and makes user interfaces more appealing and approachable for a wide variety of users. Although the current conversational interfaces are still simple, we can expect that they will integrate several features of human conversations in the future.

On the other hand, in multi-agent systems, it is widely recognized that communication between autonomous agents is a challenging research area [9, 13]. In this domain, in order to enable agents to negotiate, to solve conflicts of interest, to cooperate, to find proofs, etc., they have to be able not only to exchange single messages, but also to take part and to engage in coherent conversations with other agents as well as with human users.

In the last few years, different research works on agent communicational models based on commitments $[2,3,10,15,19,24,25,30]$ and dialogue games $[12,20,21]$ seem to offer an interesting direction. However, not only the semantics of such models are not yet standardized but also to our knowledge, none of them integrate features found in human conversations. 
The phenomena of human conversations we are interested in are those proposed as an enrichment of the traditional version of speech act theory: (1) Taking into account the non literal level of speech acts $[8,11,28]$; (2) Modeling the dialogization process (or conversational sequencing) $[8,26,29]$ and (3) Taking into account the influence of social relationships [5].

More specifically, we think that future agent/user and agent/agent interactions should allow the manipulation of indirect speech acts that are commonly used in human conversations. In addition, agents involved in such conversations should also be able to take into account the conversational sequencing and the influence of social relationships.

To illustrate the problem, let us consider the following dialogue between a human user and his conversational agent.

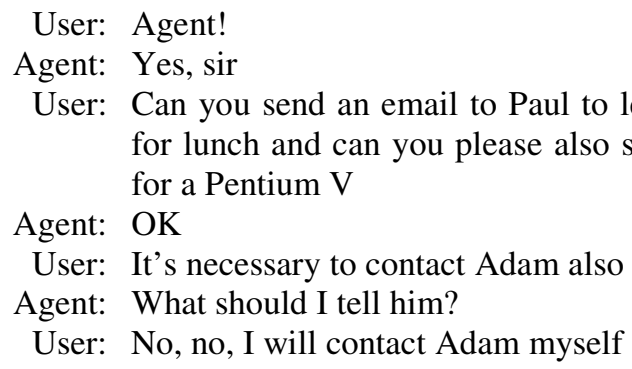

It is easily observed from this simple dialogue that an agent involved in agent/user conversation should reason on:

1. The indirectness of speech acts: can you send an email to Paul?

2. The dialogization process: the utterance "it's necessary to contact Adam" is interpreted first by the agent as a directive until the user corrects this situation later in the conversation by telling "no, no, I will contact Adam myself".

This paper is a continuation of our prior research $[1,2,3,6,7]$ that deals with the automation of conversations between human agents and software agents as well as between software agents. In this paper, we focus on two conversational phenomena: indirect speech acts and the dialogization process. More specifically, our aim is to propose an agent communicational model with its specific semantics that integrates these two phenomena of human conversations. The purpose is to show that our formal framework for social commitments can be used as a theoretical background for this model.

The paper is organized as follows. Section 2 presents the theoretical background of our approach. Section 3 introduces our communicational model. In Section 4, we see how this model deals with and manages indirect speech acts. Section 5 concerns the dialogization process. In Section 6 we describe the POSTAGE prototype. Finally, we conclude the paper and present some directions for future research.

${ }^{1}$ Simply speaking, dialogization concerns the understanding of the communicative intention between the interlocutors during the dialogue. 


\section{Theoretical Background}

\subsection{Social Commitments}

Our communication model is based on the notion of social commitments. A social commitment is a commitment made by an agent (the debtor), that some fact is true or to do something. This commitment is directed to a set of agents (creditors) [24]. Social commitments are a kind of deontic concept. They can be viewed as a generalization of obligations as studied in deontic logic [25]. Indeed, considering their deontic nature, these commitments define constraints on the agents' behavior. The agent must behave in accordance to its commitments. For example, by committing towards other agents that a certain fact is true, the agent is compelled not to contradict itself during the conversation. It must also be able to explain, argue, justify and defend itself if another participant contradicts it. In fact, we do not speak here about the expression of a belief, but rather about a particular relationship between a participant and a statement.

In our framework, the commitment content is characterized by time $t_{\varphi}$, which is different from the utterance time denoted $t_{u}$ and from the time associated with the commitment and denoted $t_{s c}$. Time $t_{s c}$ refers to the time during which the commitment holds. Fig. 1 illustrates the relation between $t_{\varphi}, t_{u}, t_{s c}$.

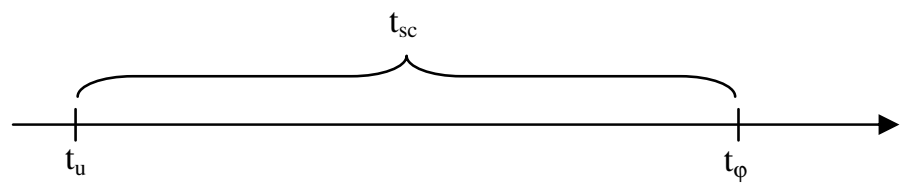

Fig. 1. Times tu, tsc and t $\varphi$

We denote a social commitment: $S C\left(A g_{1}, A^{*}, t_{s c}, \varphi, t_{\varphi}\right)$ where $A g_{1}$ the debtor, $A^{*}$ the set of the creditors $\left(A^{*}=A /\left\{A g_{1}\right\}\right)$, where $A$ is the set of participants), $t_{s c}$ is the time associated with the commitment, $\varphi$ its content and $t_{\varphi}$ the time associated with the content $\varphi$. To simplify the notation, we suppose throughout this paper that $A=\left\{A g_{1}, A g_{2}\right\}$.

In our approach we interpret a speech act as an action performed by an agent on a commitment in order to model the dynamics of conversations. This interpretation is denoted:

Definition 1. $S A\left(A g_{1}, A g_{2}, t_{u}, U\right)={ }_{\operatorname{def}} A c t\left(A g_{1}, t_{u}, S C\left(A g_{1}, A g_{2}, t_{s c}, \varphi, t_{\varphi}\right)\right)$

where $=_{\text {def }}$ means "is interpreted by definition as", $S A$ is the abbreviation of "Speech Act", and Act indicates the action performed by the debtor on the commitment. The definiendum $\left(S A\left(A g_{1}, A g_{2}, t_{w}, U\right)\right)$ is defined by the definiens ( $A c t\left(A g_{1}, t_{w}, S C\left(A g_{1}\right.\right.$, $\left.\left.\left.A g_{2}, t_{s c}, \varphi, t_{\varphi}\right)\right)\right)$ as an action performed on a commitment. The agent that performs the speech act is the same agent that performs the action Act. Act can take one of four values: Create, Withdraw, Violate and Fulfill. These four actions are the actions that the debtor can apply to a commitment. This reflects only the debtor's point of view. However, we must also take into account the creditors when modeling a conversation 
which is, by definition, a joint activity. We thus propose modeling the creditors' actions which do not apply to the commitment, but to the content of this commitment. The semantics associated with this type of actions is expressed in a dynamic logic [3]. This semantics is different from the temporal semantics proposed in [19, 25 and 30] and from the operational specification proposed in [15]. Unlike these semantics, our semantic differentiates commitments as static structures from the operations applied to these commitments as dynamic structures. In our framework, all communicative acts are actions that agents apply to commitments. This enables us to describe more naturally the evolution of the conversations as a system of states / transitions which reflects the interaction dynamics. Hence we redefine a speech act as follows:

Definition 2. $S A\left(A g_{1}, A g_{2}, t_{w}, U\right)={ }_{d e f}$

$$
\begin{aligned}
& A c t\left(A g_{1}, t_{u}, S C\left(A g_{1}, A g_{2}, t_{s c}, \varphi, t_{\varphi}\right)\right) \\
& \mid \operatorname{Act} \text {-content }\left(A g_{k}, t_{u}, S C\left(A g_{i}, A g_{j}, t_{s c}, \varphi, t_{\varphi}\right)\right)
\end{aligned}
$$

where $i, j \in\{1,2\}$ and $(k=i$ or $k=j)$. Agent $A g_{k}$ can thus act on the content of its own commitment (in this case we get $k=i$ ) or on the content of the commitment of another agent (in this case we get $k=j$ ).

For example, the utterance:

$$
U \text { : "I met agent } \mathrm{Ag}_{3} \text { on MSN one hour ago" }
$$

leads to the creation of the commitment:

$$
S C\left(A g_{1}, A g_{2}, t_{s c}, \operatorname{Meet}\left(A g_{1}, A g_{3}, M S N\right), t_{s c}-1 h\right) \text {. }
$$

The creation of such a commitment is an action denoted:

$$
\text { Create }\left(A g_{1}, t_{w}, S C\left(A g_{1}, A g_{2}, t_{s c}, \operatorname{Meet}\left(A g_{1}, A g_{3}, M S N\right), t_{s c}-1 h\right)\right) \text {. }
$$

\subsection{Taxonomy}

In this section, we explain the various types of social commitments we use in our model:

A. Absolute Commitments $(A B C)$ : They are commitments whose fulfillment does not depend on any particular condition. Two types can be distinguished:

A1. Propositional Commitments (PCs): They are related to the state of the world and expressed by assertives.

A2. Action Commitments $(A C)$ : They are always directed towards the future and are related to actions that the debtor is committed to carrying out. This type of commitments is typically conveyed by promises.

B. Conditional Commitments (CC): In several cases, agents need to make commitments not in absolute terms but under given conditions. Conditional commitments allow us to express that if a condition $\beta$ is true, then the creditor will be committed towards the debtor to making $\gamma$ or that $\gamma$ is true.

C. Commitment Attempts (CT): The social commitments described so far directly concern the debtor who commits either that a certain fact is true or that a certain action will be carried out. These commitments do not allow us to explain the fact that an agent asks another one to be committed to carrying out an action. To solve this problem, we propose the concept of commitment attempt. We consider a commitment attempt as a request made by a debtor to push a creditor to be committed. 


\section{The Communicational Model}

Computationally speaking, a conversational model should possess a communicational model to which we integrate the phenomena we are interested in. Our communication model is based on the following fundamental principles:

- Communication is considered as a negotiation process [17, 23]. This process is formed by a set of initiative/reactive dialogue games [12, 21].

- Communication results in a manipulation of social commitments [1, 10, 20, 24].

- Agents use their private mental states to manipulate social commitments.

We adopt these principles in our approach and we consider agents' communication as actions applied on commitments and as exchanges of what we call communicational states $^{2}$ (CS). Fig. 2 illustrates our communication model.

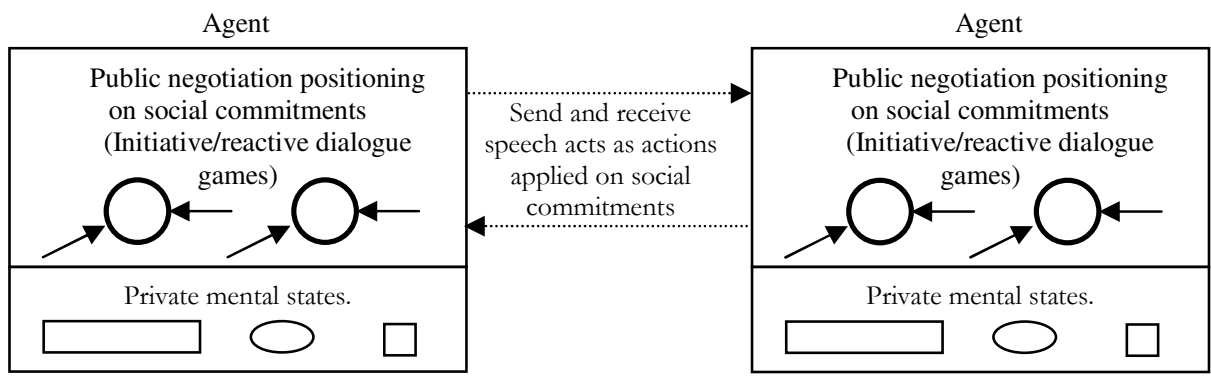

Fig. 2. The communicational model

A CS is characterized by one of five types, each type corresponding to a performative type as defined by Vanderveken [27]. Since we consider that agents communicate by conversing, a CS is similar to a speech act and is used by an agent to express its communicative intentions. However, a CS differs from a speech act in the sense that (1) a CS is associated to a negotiation positioning and (2) a CS is not composed of seven components as it is the case for a speech act [27]. A CS is also expressed in terms of social commitments.

A directive CS performed by an agent $A g_{1}$ toward an agent $A g_{2}$ at time $t$ concerning the propositional content $p$ has the following form ${ }^{3}: \operatorname{DIR}\left(\mathrm{Ag}_{1}, A g_{2}, t, p, t_{p}\right)$ where $t_{p}$ is the time associated to the content $p$. A directive CS is defined as a commitment attempt:

Definition 3. $\operatorname{DIR}\left(A g_{1}, A g_{2}, t, p, t_{p}\right)=_{d e f} C T\left(A g_{1}, A g_{2}, t_{c}, p, t_{p}\right)$

where $t=t_{c t}$.

The other types of CS are ASS for an assertive, DECL for a declarative, COMMIT for a commissive and EXPR for an expressive. To represent explicitly the conditional aspect of assertives and commissives, we add two other types of CS: CON-ASS for

\footnotetext{
${ }^{2}$ We chose the term "Communicational State" analogously to the term "Mental State".

${ }^{3}$ For the formalization of communicational states, we have been inspired by the work of [13].
} 
conditional assertives and CON-COMMIT for conditional commissives. An assertive CS is defined as a propositional commitment and a conditional assertive is defined as a conditional commitment about a proposition (Definition 4). A declarative and an expressive CS are defined as propositional commitments (Definitions 5 and 6). Finally, a commissive CS is defined as an action commitment and a conditional commissive is defined as a conditional commitment about an action (Definition 7).

Definition 4. $A S S\left(A g_{1}, A g_{2}, t, p\right)={ }_{d e f} P C\left(A g_{1}, A g_{2}, t_{p c}, p, t_{p}\right)$

$$
C O N-A S S\left(A g_{1}, A g_{2}, t, p_{1}, t_{p 1}, p_{2}, t_{p 2}\right)={ }_{d e f} C C\left(A g_{1}, A g_{2}, t_{c c}, p_{1}, t_{p 1}, p_{2}, t_{p 2}\right)
$$

Definition 5. $D E C L\left(A g_{1}, A g_{2}, t, p, t_{p}\right)=_{d e f} P C\left(A g_{1}, A g_{2}, t_{p c}, p, t_{p}\right)$

Definition 6. $\operatorname{EXPR}\left(A g_{1}, A g_{2}, t, p, t_{p}\right)=_{d e f} P C\left(A g_{1}, A g_{2}, t_{p c}, p, t_{p}\right)$

Definition 7. COMMIT( $\left.A g_{1}, A g_{2}, t, \alpha, t_{\alpha}\right)=_{\text {def }} A C\left(A g_{1}, A g_{2}, t_{a c}, \alpha, t_{\alpha}\right)$ $C O N-C O M M I T\left(A g_{1}, A g_{2}, t, p, t_{p}, \alpha, t_{\alpha}\right)=_{d e f} C C\left(A g_{1}, A g_{2}, t_{c c}, p, t_{p}, \alpha, t_{\alpha}\right)$

where $p$ is a propositional formula and $\alpha$ is an action symbol.

Communication is considered as a set of initiative/reactive dialogue games in which agents negotiate about CSs. In other words, agents negotiate the acceptance or the refusal of CSs. An agent proposes a CS (initiative dialogue game) and other agents react to this proposal by accepting, rejecting the proposed CS, asking for further information, etc. (reactive dialogue game). Thus, a negotiation positioning is associated to a CS. Since finding a settlement is not the main goal of our negotiation process, this process is different from the negotiation dialogue defined in Walton and Krabbe's typology [31]. On the other hand, this process is similar to the persuasion dialogue that arises from a conflict of opinions and whose goal is to solve the conflict. In our framework, a positioning takes the following form:

$$
\operatorname{POSIT}\left(A g_{1}, A g_{2}, t, C S\left(A g_{1}, A g_{2}, t, \varphi, t_{\varphi}\right)\right)
$$

where $C S \in\{D I R, A S S, D E C L, E X P R, C O M M I T, C O N D-C O M M I T\}$

which represents the positioning of agent $A g_{1}$ toward agent $A g_{2}$ at time $t$ with respect to a communicational state $C S$.

The positionings we consider are the proposition PROPOSE, the acceptance $A C C E P T$ and the refusal REFUSE of a CS. We also add the special INQUIRE positioning for asking questions. We distinguish two types of INQUIRE. The first type requires a $\mathrm{Yes} / \mathrm{No}$ answer. The second type requires an answer substituting a set of free variables $X$ in the propositional content by a certain valuation. We denote a formula $\varphi$ in which appears a sequence of free variables $X$ by ?X $\varphi$. These two types of INQUIRE are denoted as follows:

$$
\begin{aligned}
& \operatorname{INQUIRE}\left(A g_{1}, A g_{2}, t, C S\left(A g_{1}, A g_{2}, t, p, t_{p}\right), Y e s / N o ?\right) \\
& \operatorname{INQUIRE}\left(A g_{1}, A g_{2}, t, C S\left(A g_{i}, A g_{j}, t, ? X \varphi, t_{\varphi}\right)\right)
\end{aligned}
$$

where $i, j \in\{1,2\}$ and $i \neq j$.

A positioning with respect to a communicational state $C S$ is defined as an action applied by an agent on a social commitment SC or on the content of a social commitment: 
Definition 8. $\operatorname{POSIT}\left(A g_{1}, A g_{2}, t, C S\left(A g_{1}, A g_{2}, t, \varphi, t_{\varphi}\right)\right)=_{d e f}$

$$
\begin{aligned}
& \operatorname{Act}\left(A g_{1}, t_{u}, S C\left(A g_{1}, A g_{2}, t_{s c}, \varphi, t_{\varphi}\right)\right) \\
& \mid \operatorname{Act} \text {-content }\left(A g_{k}, t_{u}, S C\left(A g_{i}, A g_{j}, t_{s c}, \varphi, t_{\varphi}\right)\right)
\end{aligned}
$$

where $i, j \in\{1,2\}$ and $(k=i$ or $k=j)$.

For example, the proposition of a CS is defined as a creation action of a commitment (Definition 9). The commitment type depends on the type of the CS as specified by Definitions 4, 5, 6 and 7.

Definition 9. $\operatorname{POSIT}\left(A g_{1}, A g_{2}, t_{w}, C S\left(A g_{1}, A g_{2}, t, \varphi, t_{\varphi}\right)\right)={ }_{d e f}$

$$
\text { Create }\left(A g_{1}, t_{u}, S C\left(A g_{1}, A g_{2}, t_{s c}, \varphi, t_{\varphi}\right)\right)
$$

Let us take the following simple dialogue between agents $A g_{1}$ and $A g_{2}$.

$$
\begin{array}{lll}
\left(S A_{1}\right) & A g_{1}: & \text { Print the document number } 5 \\
\left(S A_{2}\right) & A g_{2}: & O k !
\end{array}
$$

The speech act $S A_{l}$ is represented by the proposal of a directive:

$$
\operatorname{PROPOSE}\left(\mathrm{Ag}_{1}, \mathrm{Ag}_{2}, \mathrm{t}_{1}, \mathrm{CS}_{1}\right)
$$

where $C S_{1}$ represents $\operatorname{DIR}\left(A g_{1}, A g_{2}, t_{1}, \operatorname{print}\left(A G T\left(A g_{2}\right), O B J(\right.\right.$ document-5)))

$A g_{1}$ is proposing to $A g_{2}$, at time $t_{l}$, a directive in which $A g_{1}$ is asking $A g_{2}$ at time $t_{l}$ that agent $A g_{2}$ print the object document 5. The speech act $S A_{2}$ is represented by the acceptance of the first directive:

$$
A C C E P T\left(A g_{2}, A g_{1}, t_{2}, C S_{1}\right)
$$

$A g_{2}$ is accepting, at time $t_{2}$, the directive proposed at time $t_{1}$ where $A g_{1}$ is asking $A g_{2}$ to print the document 5 .

Furthermore, it is easy to notice that usually human conversants are able to recall the utterances (at least the most important ones) that have been exchanged during a conversation along with the locutors' positionings. In our approach, we consider that the exchanged CSs are recorded into a conceptual structure called the conversational trace. Using the conversational traces of both agents, this dialogue is represented in Fig. 3. It is important to mention that each agent possesses its own conversational trace and thus its own viewpoint of the communication. This assumption of no central agent (called also external observer) is considered in other agent models. It is the case

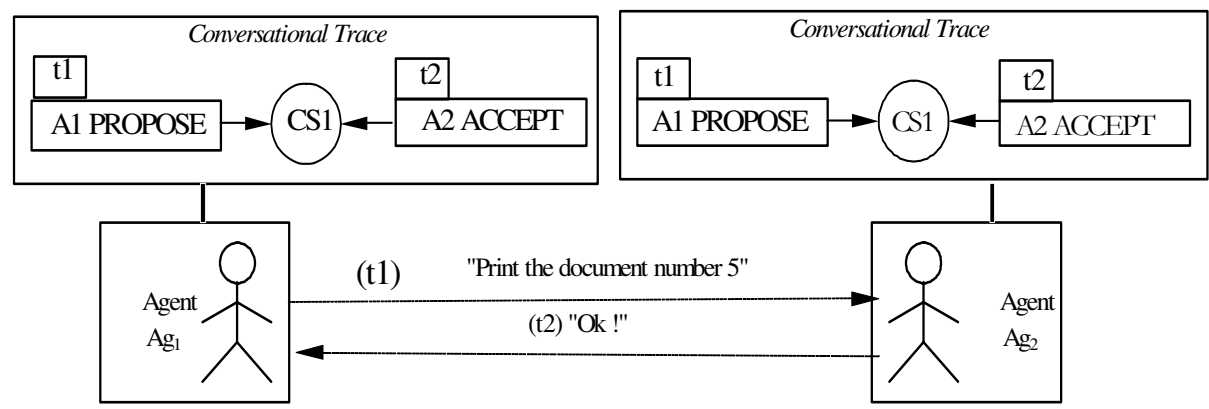

Fig. 3. Conversational traces of agents $\mathrm{Ag}_{1}$ and $\mathrm{Ag}_{2}$ 
for [4] where agents detect ontological discrepancies during communication on the basis of their own subjective view on the world.

Our communicational model is based on a negotiation process in which agents (human or artificial) are negotiating on CSs. Agents record all the negotiation positionings as well as the CSs during the conversation process. Let us see now how this model can be used in order to deal with the non-literal level of speech acts.

\section{Implicit Information}

It is easily observed that human locutors use indirect speech acts more frequently than direct speech acts. For instance, when a manager says to his secretary "Can you print the document number 5?", his utterance should be interpreted as a polite way of ordering her to print the document (non literal interpretation) and not as a question about her ability to print (literal interpretation). Also, the question asked by the user to his agent (in the first dialogue) "Can you send an email to Paul?" should be interpreted too as a directive speech act.

In order to take into account this conversational phenomenon, we suggested to model implicit information conveyed by speech acts [6]. Given a speech act $S A$ performed by locutor $L_{1}$ and directed to locutor $L_{2}$, we define the implicit information conveyed by $S A$ as the information that $L_{1}$ intends to transfer to $L_{2}$ and which is different from $S A$ 's propositional content. For example, the implicit information associated with the question "Can you send an email to Paul?" is the request to send the email. To our knowledge, no current implementation of software agents integrates this aspect in its communicational model. Implicit information can be compared to presuppositions that Beun et al. [4] are using in their model. Indeed, in that model, agents extract presuppositions from incoming messages on the basis of the pragmatics of the communication language.

In order to provide a mapping between implicit and explicit information, we use knowledge structures called conversational schemas that are similar to conversational postulates that Gordon and Lakoff proposed to interpret indirect speech acts [16]. Conversational schemas specify conversational conventions that apply in a given socio-organizational context. A conversational schema can be used by an agent either for choosing a speech act that reflects its communicative intention, or for interpreting other agents' speech acts. For example, the conversational schema of the above example could be formulated by the following definition:

Definition 10: INQUIRE $\left(A g_{1}, A g_{2}, t, C S\left(A g_{1}, A g_{2}, t\right.\right.$,

$$
\begin{gathered}
\left.H A S-C A P A C I T Y\left(A G T\left(A g_{2}\right), O B J(P r o p)\right), Y e s / N o ?\right)={ }_{d e f} \\
P R O P O S E\left(A g_{1}, A g_{2}, t, D I R\left(A g_{1}, A g_{2}, t, \text { Prop }\right)\right)
\end{gathered}
$$

A conversational schema has the following form:

CONV-SCH "ident"
Context
Characteristics
Communicative intention
Explicit information
Communicative Expectation


Each agent possesses a set of conversational schemas. This set represents its knowledge of the conversational practices of the society to which it belongs. The set of conversational schemas that agents share could be considered as part of the common conversational ground of these agents. The Characteristics slot has two components. The first component concerns the illocutionary strength, which is quantitative, and allows the agent to have different formulations for the same communicative intention. The second component is the refusal option that indicates if the agent can refuse a given directive. When an agent wants to express a certain Communicative intention, it chooses a conversational schema depending on the social and personality context. This conversational schema gives it the corresponding formulation in the Explicit information slot. The slot Communicative Expectation will be explained in the next section.

For example, the corresponding conversational schema for a "polite request" is formulated as follows:

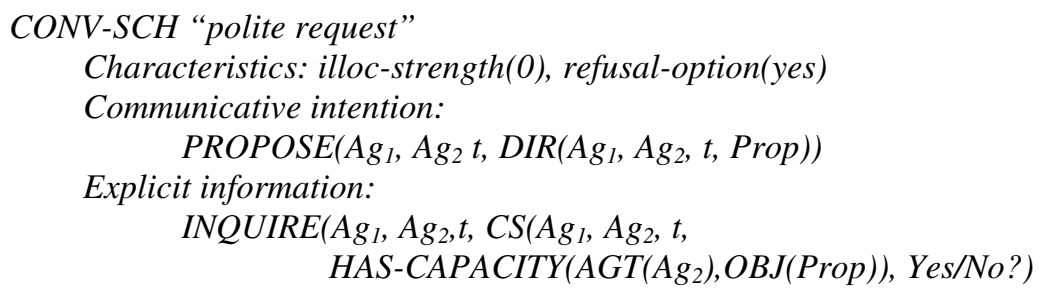

The above "polite request" conversational schema is used by an agent $A g_{1}$ toward an agent $\mathrm{Ag}_{2}$.. This conversational schema has illocutionary strength of 0 and it concerns a directive CS $(D I R)$, which gives a refusal option to the interlocutor. In this $\mathrm{CS}$, agent $A g_{1}$ has the intention to propose a directive to agent $A g_{2}$ and for this purpose, it will publicly perform an inquire (INQUIRE) asking agent $A g_{2}$ about its capacity to do the needed action expressed by Prop. Indeed, explicit information indicates the action applied by the agent on a social commitment.

In order to take into account the explicit and implicit information managed by an agent during a dialogue, we divided the conversation trace into two categories: an explicit conversational trace in which it records the public utterances and an implicit conversational trace in which it records the intentional utterances. This aspect is detailed in the next section. Let us mention that we plan to extend our approach in order to take into account the influence of social relationships during the interaction. Indeed, according to [5], there is little doubt that social relationships influence the way people interpret indirect speech acts. Some preliminary results of this extension could be found in [6].

\section{Dialogization}

Dialogization is based on the understanding of the communicative intention between interlocutors during the dialogue. In other agent frameworks [4], this phenomenon is called feedback. The schema of the dialogization process is shown in Fig. 4. During the first stage, an initiator agent $A g_{1}$ makes an initial proposal corresponding to its communicative intention. It waits for the positioning of its interlocutor agent $A g_{2}$ 
regarding this proposal. If $\mathrm{Ag}_{2}$ 's positioning matches what $A g_{1}$ was expecting as an answer, then it concludes that $A g_{2}$ understood its communicative intention and in this case it can go ahead and make another proposal. In the case in which $\mathrm{Ag}_{2}$ 's positioning doesn't match what $A g_{1}$ is expecting, then it concludes that $A g_{2}$ didn't understand its communicative intention and reacts by expressing its communicative intention more explicitly. This is done by the choice of a different conversational schema in which the communicational intention and explicit information slots are almost the same.

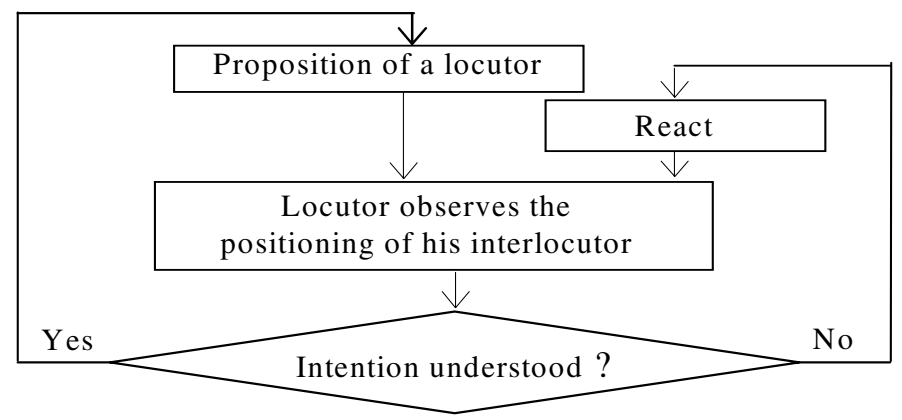

Fig. 4. The dialogization process

We still need to provide a way to determine if the agent's intention has been understood or not by its interlocutor. This is the role of the Communicative Expectation slot of a conversational schema. In our approach, an agent $A g_{l}$ determines if the intention corresponding to its initial speech act has been recognized by the interlocutor agent $\mathrm{Ag}_{2}$, if $\mathrm{Ag}_{2}$ 's speech act matches the Communicative Expectation. For instance, the actual "polite request" conversation schema is:

CONV-SCH "polite request"

Characteristics: illoc-strength( 0 ), refusal-option(yes)

Communicative intention:

$\operatorname{PROPOSE}\left(A g_{1}, A g_{2}, t_{1}, \operatorname{DIR}\left(A g_{1}, A g_{2}, t_{1}\right.\right.$, Prop $\left.)\right)$

Explicit information:

$\operatorname{INQUIRE}\left(A g_{1}, A g_{2}, t_{l}, C S\left(A g_{1}, A g_{2}, t_{1}\right.\right.$, HAS-CAPACITY (AGT( $\left.\left.\mathrm{Ag}_{2}\right), \mathrm{OBJ}(\mathrm{Prop})\right)$, Yes/No?)

Communicative Expectation:

$\operatorname{ACCEPT}\left(A g_{2}, A g_{1}, t_{2}, \operatorname{DIR}\left(A g_{1}, A g_{2}, t_{1}\right.\right.$, Prop $\left.)\right)$

This conversational schema states that $A g_{1}$ will expect $A g_{2}$ to accept its proposal of the implicit directive even if $A g_{1}$ publicly asks $\mathrm{Ag}_{2}$ about its capacity of doing the needed action (represented by Prop).

Let us take as an example the following dialogue illustrating the dialogization phenomenon. 
(1) User: It's necessary to contact Adam

(2) Agent: What should I tell him?

(3) User: No, no, I will contact Adam myself

(4) Agent: $O K$ !

The agent interpreted the first user's utterance as a directive, while the user actually intended only to express an assertive. The agent responded to the directive by asking information about the way to execute it. The user expecting an acceptance of the assertive, reacts to this question, and expresses his assertive more explicitly. The corresponding conversational traces - both implicit and explicit - of the user are illustrated in Fig. 5. An oval shape represents a CS. Using a plain line,

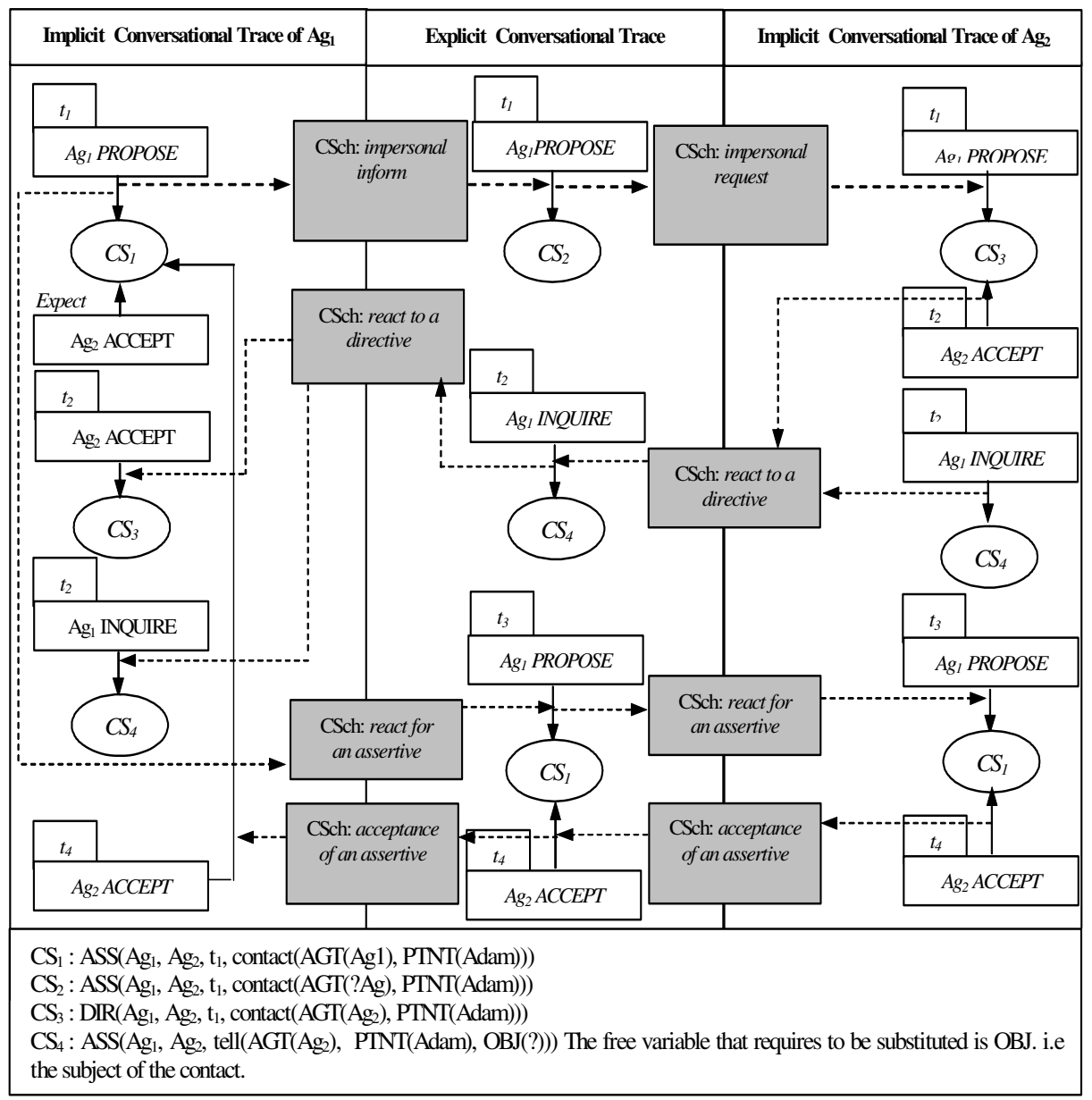

Fig. 5. Implicit and explicit conversational traces of agents $\mathrm{Ag}_{1}$ and $\mathrm{Ag}_{2}$ 
each CS is associated to a rectangular shape representing a given position with its time point (e.g. $A g_{1}$ PROPOSE $C S_{1}$ at $t_{l}$ ). Using dashed lines, the negotiation positioning (position and CS) are associated to gray background rectangles that represent the Conversational Schema the user is using to interpret the negotiation positioning (e.g. $\mathrm{Ag}_{1} \quad$ PROPOSE $\mathrm{CS}_{2}$ at $t_{1} \rightarrow$ use of ConvSc (impersonal inform $) \rightarrow A g_{l}$ PROPOSE $C S_{1}$ at $\left.t_{l}\right)$.

At time $t_{l}, A g_{l}$ wants, at the implicit level, to propose an assertive $A S S C S_{l}$ consisting of contacting Adam. This is represented by the structure:

$\operatorname{PROPOSE}\left(A g_{1}, A g_{2}, t_{1}, A S S\left(A g_{1}, A g_{2}, t_{l}, \operatorname{contact}\left(A G T\left(A g_{1}\right), \operatorname{PTNT}(\operatorname{Adam})\right)\right)\right)$

Using the conversational schema 'impersonal inform', the user $A g_{1}$ translates the proposition of $C S_{1}$ to a proposition of another assertive $C S_{2}$ in which the agent is unknown. This assertive that becomes public has the structure:

$\operatorname{PROPOSE}\left(\mathrm{Ag}_{1}, A g_{2}, t_{1}, \operatorname{ASS}\left(\mathrm{Ag}_{1}, A g_{2}, t_{1}, \operatorname{contact}(A G T(? A)), \operatorname{PTNT}(\right.\right.$ Adam $\left.\left.\left.)\right)\right)\right)$

At the same time, $A g_{1}$ expects from $A g_{2}$ to accept $C S_{1}$ :

$A C C E P T\left(A g_{2}, A g_{1}, t_{2}, A S S\left(A g_{1}, A g_{2}, t_{1}, \operatorname{contact}\left(A G T\left(A g_{1}\right), P T N T(A d a m)\right)\right)\right)$

Using the conversational schema 'impersonal request', agent $A g_{2}$ who receives this proposition determines in its implicit conversational trace that $A g_{1}$ is requesting it to contact Adam $\left(\mathrm{CS}_{3}\right)$. At time $t_{2}, A g_{2}$ implicitly accepts the directive and publicly asks $A g_{1}$ how to do that action, using the conversational schema 'react to a directive'. At this time, $A g_{1}$ observes that $A g_{2}$ is accepting a directive but not an assertive:

$$
\operatorname{ACCEPT}\left(\mathrm{Ag}_{2}, A g_{1}, t_{2}, \operatorname{DIR}\left(\mathrm{Ag}_{1}, A g_{2}, t_{1}, \operatorname{contact}\left(A G T\left(A g_{2}\right), \operatorname{PTNT}(\operatorname{Adam})\right)\right)\right)
$$

$A g_{1}$ understands that $A g_{2}$ used the conversational schema 'impersonal request' to infer $C S_{3}$ from $C S_{2}$. Thus, what $A g_{1}$ is expecting does not match the answer of $A g_{2}$. This leads $A g_{1}$ to react in order to state his first communicative intention. He uses for this purpose, at time $t_{3}$, a more explicit conversational schema 'react for an assertive':

$$
\operatorname{PROPOSE}\left(A g_{1}, A g_{2}, t_{3}, A S S\left(A g_{1}, A g_{2}, t_{3}, \operatorname{contact}\left(A G T\left(A g_{1}\right), \operatorname{PTNT}(\text { Adam) })\right)\right)\right.
$$

At time $t_{4}, A g_{2}$ accepts $C S_{1}$ implicitly and publicly by using the conversational schema 'acceptance of an assertive'. The acceptance of $A g_{2}$ means for $A g_{1}$ that $A g_{2}$ understood the intention since the answer matches the communicative expectation:

$$
\operatorname{ACCEPT}\left(A g_{2}, A g_{1}, t_{4}, A S S\left(A g_{1}, A g_{2}, t_{3}, \operatorname{contact}\left(A G T\left(A g_{1}\right), \operatorname{PTNT}(\operatorname{Adam})\right)\right)\right)
$$

In this section, we explained how the dialogization process can be modeled using our model. Indeed, adding a communicative expectation to a conversational schema allows agents to reason on that process: they compare their interlocutors' positioning with their expectation and react accordingly.

The time complexity of the algorithm implementing this process is linear in the size of the communicative intention bases $\left|C B_{A g l}+C B_{A g 2}\right|$ that are a kind of the knowledge bases of the two agents. It is also linear with the number $N C S_{A g 1}+$ $\mathrm{NCSAg}_{2}$ of the conversational schemas that the two agents can use. Because we associate to each communicative intention $n$ conversational schemas $(n \geq 1)$, the time complexity is only linear in the number of the conversational schemas, i.e. $\mathrm{O}\left(\max \left(N C S_{A g 1}, N C S_{A g 2}\right)\right)$. 


\section{The POSTAGE Prototype}

In large and small organizations, correspondence between users exists in various forms: formal and informal letters, memos, notices, etc. Developing a software agent taking care of the administrative correspondence would greatly benefit to the user: (1) the user is not obliged to remember all the formulations used in his/her organization thanks to the use of conversational schemas; (2) The user can be informed about the different interpretations of a message thanks to the dialogization process done by the agent.

The POSTAGE (POSTman AGEnt) agent can formulate a user's message in an informal way which agrees with (1) the user communicative intention and (2) the formulation rules used in a particular organization. For example, the informal message "You are laid off" would be transformed into "As general manager, I deeply regret having to announce your dismissal from our company". For the present work, we have chosen the university organization as an example for the development of the prototype. A POSTAGE agent has a specific architecture that allows it to perform the correspondence task (Fig. 6). This architecture is divided into two parts. The first part includes four knowledge models and the second one three execution modules. The user's model contains knowledge concerning the user such as his/her preferences and his/her social relationships with other users. The static knowledge contains plans and specific formulation schemas. A formulation schema is used by the agent to find a natural language expression for a given conversational schema.

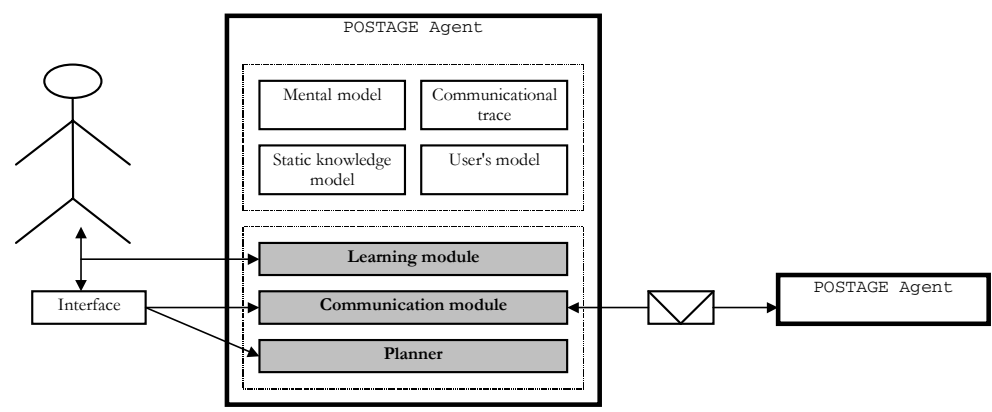

Fig. 6. Architecture of a POSTAGE agent

The other modules are the communicational trace and the mental model. The planning module allows the agent to create messages on the basis of the elements selected by the user. The task of the learning module is to learn new knowledge such as user's preferences or formulations used in a given organization. The communication module receives a request from the planning module and determines the corresponding negotiation positionings.

Let us show an example using the conversational sequencing reasoning of the POSTAGE agent (Fig. 7). Consider two users Viviane and Brigitte with their corresponding agents. Viviane sends a first message to Brigitte by selecting as a subject "Update of web pages". Viviane clicks on the generate button and the agent proposes the text "Can you please update the web pages?". When Brigitte's agent 
receives the message, it notices that this text has two possible interpretations, and asks Brigitte the interpretation she prefers. In this example, Brigitte takes the literal interpretation. When Viviane's agent receives the answer, it automatically understands that the answer does not correspond to Viviane expectation, informs her, and proposes her to re-express her intention more explicitly. In this case, Viviane's agent proposes the text "No, I am asking you to update the web pages".

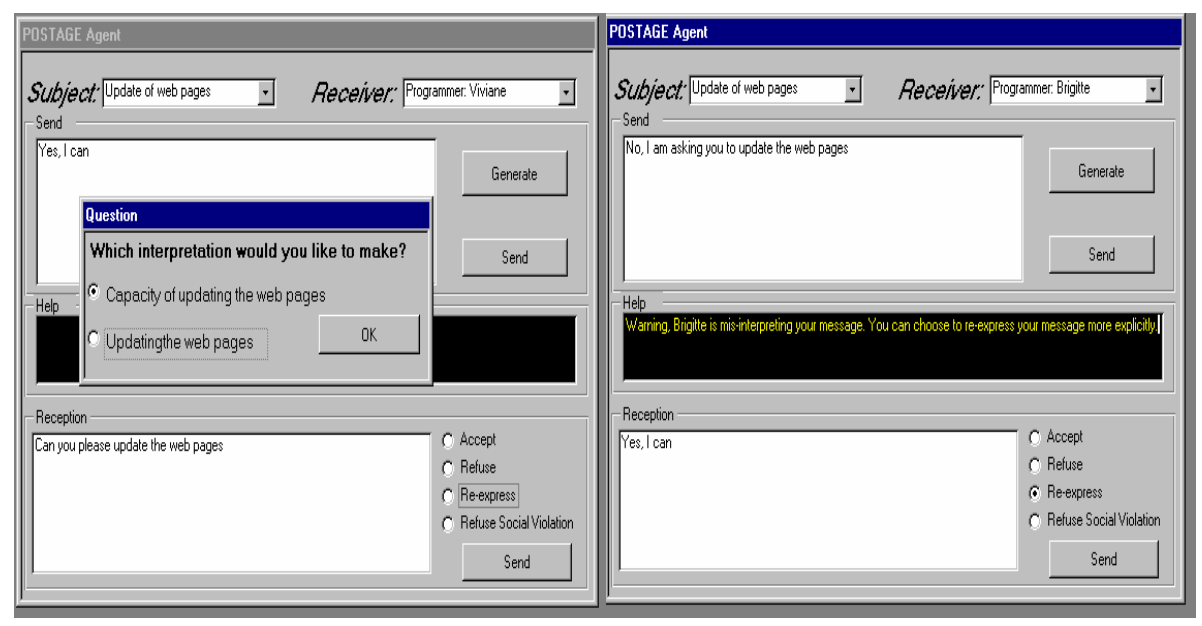

Fig. 7. The conversational sequencing

\section{Conclusion and Future Work}

In this paper we proposed a computational model for human-agent and agent-agent conversation. This model deals with the implicit aspects of conversations and the dialogization process. The implicit aspects is captured by taking into account the non literal level of speech acts. The dialogization process is treated by considering communication as a negotiation process of social commitments. This process is formed by a set of initiative/reactive dialogue games.

As future work, we intend to integrate the influence of social relationships in our framework and to improve our prototype by using real corpora. We also intend to integrate argumentation issues to capture the reasoning aspect of agents.

\section{References}

1. Bentahar, J., Moulin, B., and Chaib-draa, B.: Vers une approche à base d'engagements et d'arguments pour la modélisation du dialogue. Modèles Formels de l'Interaction (2003) 19-28.

2. Bentahar, J., Moulin, B., and Chaib-draa, B.: Commitment and Argument Network: a New Formalism for Agent Communication. In: F. Dignum (Ed.) Advances in Agent Communication. LNAI 2922. Springer (2004) 146-165. 
3. Bentahar, J., Moulin, B., Meyer, J-J. Ch., and Chaib-draa, B.: A logical model for commitment and argument network for agent communication. In: Proc. Of the 3rd Int. J. Conf. On AAMAS (2004) 792-799.

4. Beun, R.J., Van Eijk, R.M., Prüst, H., Ontological Feedback in Multiagent Systems, In: Proc. Of the 3rd Int. J. Conf. On AAMAS (2004), 110-117.

5. Boden, D., and Zimmerman, D.H.: Talk and social structures. Berkley: University of California Press (1991).

6. Bouzouba, K., Moulin, B.: KQML+: An extension of KQML in order to deal with implicit information and social relationships. In: Proc. of FLAIRS'98 Sanibel Island (1998) 289293.

7. Bouzouba, K., Moulin, B., and Kabbaj, A.: CG-KQML+: An Agent Communication Language and its use in a Multi-Agent System. In: Proc of the 9th Int. Conf. on Conceptual Structures (2001) 1-14.

8. Brassac, C.: Speech acts and conversational sequencing. Pragmatics and cognition. Vol. 2(1) (1994) 191-205.

9. Chaib-draa, B. and Dignum, F.: Trends in agent communication language. In: Computational Intelligence. Vol. 18(2) (2002) 1-14.

10. Colombetti, M.: A commitment-based approach to agent speech acts and conversations. In: the Autonomous Agent Workshop on Conversational Policies. 4th Int. Conf. on Autonomous Agent (2000) 21-29.

11. Dascal, M.: On the pragmatic structure of conversation. In: J.R. Searle et al. (On) Searle on Conversation, compiled and introduced by H. Parret and J. Verschueren. Amsterdam: John Benjamins (1992) 35-56.

12. Dastani, M., Hulstijn, J., and van der Torre, L.: Negotiation protocols and dialogue games. In: Proc. of the Belgium/Dutch AI Conf. (BNAIC'2000) Kaatsheuvel (2000) 13-20.

13. Dignum, F. and Greaves, M.: Issues in agent communication: an introduction. In: F. Dignum, and M. Greaves (Eds.). Issues in Agent Communication. LNAI 1916. Springer (2000) 1-16.

14. Dignum, F. and van Linder, B.: Modelling social agents: Communication as actions. In: M. Wooldridge, J. Muller, and N. Jennings (Eds.). Intelligent Agents III. LNAI 1193. Springer (1997) 205-218.

15. Fornara, N. and Colombetti, M.: Operational specification of a commitment-based agent communication language. In: Proc. of the First Int. J. Conf. on AAMAS (2002) 536- 542.

16. Gordon, D. and Lakoff, G.: Conversational postulates. In: P. Cole \& J.L. Morgan (Eds.). Syntax and semantics: Vol. 3. Speech acts. Seminar Press (1975) 83-106.

17. Haddadi, A. Communication and Cooperation in Agent Systems. A Pragmatic Theory. Springer (1995).

18. Hayes-Roth, B., van Gent, R., Hubert, D.: Acting in Character. Technical Report KSL 9613. Knowledge Systems Laboratory (1996).

19. Mallya, A.U., Yolum, P., and Singh, M.: Resolving commitments among autonomous agents. In: Dignum, F. (ed.). Advances in Agent Communication. Springer (2003) 166-182.

20. Maudet, N. and Chaib-draa, B.: Commitment-based and dialogue-game based protocols, new trends in agent communication languages. In: Knowledge Engineering Review. Vol. 17(2) Cambridge University Press (2002) 157- 179.

21. McBurney, P.J. and Parsons, S.: Games that agents play: A formal framework for dialogues between autonomous agents. In: Journal of Logic, Language, and Information. 11(3) (2002) 1-22.

22. Microsoft, ActiveXTM Technology for interactive Software Agents.

23. ttp://www.microsoft.com/intdev/agent/. 
24. Rousseau, D., Moulin, B., and Lapalme, G.: A multiagent approach for modelling conversations. In: Proc. of the Int. conference AI'94. Sub-Conf. on Natural Language (1994) 35-50.

25. Singh, M.P.: Agent communication languages: rethinking the principles. IEEE Computer (1998) 40-47.

26. Singh, M.P.: A social semantics for agent communication language. In F. Dignum and M. Greaves (Eds.). Issues in Agent Communication. Springer (2000) 31-45.

27. Van Rees, M.A.: The adequacy of speech act theory for explaining conversational phenomena: a response to some conversation analytical critics. Journal of Pragmatics. 17 (1992) 31-47.

28. Vanderveken, D.: Meaning and speech acts. Vol.1 Principles of language use. Cambridge University Press (1990).

29. Vanderveken, D.: Formal Pragmatics of Non Literal Meaning. In: the Special Issue (edited by E. Rolf) Pragmatik de Linguistische Berichte. Vol 8 (1997) 324-341.

30. Vanderveken, D.: Illocutionary Logic and Discourse Typology. In: Special Issue 216 (edited by D. Vanderveken) Searle with his Replies of Revue Internationale de Philosophie (2001) 243-255.

31. Verdicchio, M. and Colombetti, M.: A logical model of social commitment for agent communication. The Second Int.. Conf. on AA MAS (2003) 528-535.

32. Walton, D.N. and Krabbe, E.C.W. Commitment in dialogue: basic concepts of interpersonal reasoning. State Univ. of New York Press, Albany, NY (1995). 